\title{
Dynamics of Saturated Energy Condensation in Two-Dimensional Turbulence
}

\author{
Chi-kwan Chan, ${ }^{1, *}$ Dhrubaditya Mitra, ${ }^{1, \dagger}$ and Axel Brandenburg ${ }^{1,2, \ddagger}$ \\ ${ }^{1}$ NORDITA, Roslagstullsbacken 23, SE-10691 Stockholm, Sweden \\ ${ }^{2}$ Department of Astronomy, Stockholm University, 10691 Stockholm, Sweden
}

(Dated: April 20, 2018)

\begin{abstract}
In two-dimensional forced Navier-Stokes turbulence, energy cascades to the largest scales in the system to form a pair of coherent vortices known as the Bose condensate. We show, both numerically and analytically, that the energy condensation saturates and the system reaches a statistically stationary state. The time scale of saturation is inversely proportional to the viscosity and the saturation energy level is determined by both the viscosity and the force. We further show that, without sufficient resolution to resolve the small-scale enstrophy spectrum, numerical simulations can give a spurious result for the saturation energy level. We also find that the movement of the condensate is similar to the motion of an inertial particle with an effective drag force. Furthermore, we show that the profile of the saturated coherent vortices can be described by a Gaussian core with exponential wings.
\end{abstract}

PACS numbers: 47.27.-i, 47.27.De, 47.27.E-

\section{INTRODUCTION}

Two-dimensional (2D) hydrodynamic turbulence is fundamentally different from its three-dimensional counterpart. In 2D, small vortices can merge to form bigger coherent vortices. This is because the equations of ideal hydrodynamics in two dimensions have, in addition to energy, also enstrophy as a conserved quantity. With an external force at an intermediate scale and viscous dissipation, energy inversely cascades to larger length scales and enstrophy directly cascades to smaller length scales [1-3].

Let us now consider 2D turbulence in a finite domain of size $L$. The smallest wave number allowed in this system is $k_{1} \equiv 2 \pi / L$. Due to the inverse cascade, energy piles up at $k_{1}$ provided there is no large-scale friction. This phenomenon, sometimes called Bose condensation in 2D turbulence (see Fig. 1), was first predicted by Kraichnan [1]. It was studied numerically by Hossain et al. [4], Smith and Yakhot [5, 6], Chertkov et al. [7] and experimentally by Paret and Tabeling [8], Xia et al. [9].

Following standard convention, we refer to the modes at $|\boldsymbol{k}|=k_{1}$ as the condensate in this paper. For a fixed non-zero viscosity, $\nu$, the energy of the condensate vortices cannot grow without limit but saturate $[10,11]$. The saturation occurs at time scales of the order of $1 / \nu k_{1}^{2}$. This is an unusual example of viscous effects playing an important role in fluid turbulence at large length scales. In this paper we show, by direct numerical simulations (DNS), that the saturation of the condensate energy is not only determined by viscosity but also by the forcing wave number $k_{\mathrm{i}}$. Furthermore, we will demonstrate that the direct enstrophy cascade must be well resolved for accurate numerical determination of the saturation.

\footnotetext{
${ }^{*}$ Electronic address: ckch@nordita.org

${ }^{\dagger}$ Electronic address: dhruba.mitra@gmail.com

${ }^{\ddagger}$ Electronic address: brandenb@nordita.org
}

Motivated by the analogy between the formation of large-scale structures in two-dimensional turbulence and the large-scale dynamo process in three-dimensional helical magnetohydrodynamic turbulence [12], we propose a simple three-scale model which is able to capture the important aspects of our numerical results. We further show that the Lagrangian dynamics of the condensate vortices can be described by Langevin equations for particles with inertia. Finally, we measure the profile of the saturated coherent vortices, which consist of the condensate and its higher harmonics. The vorticity at the cores are well fitted by a sharp Gaussian; while the wings fall off exponentially.

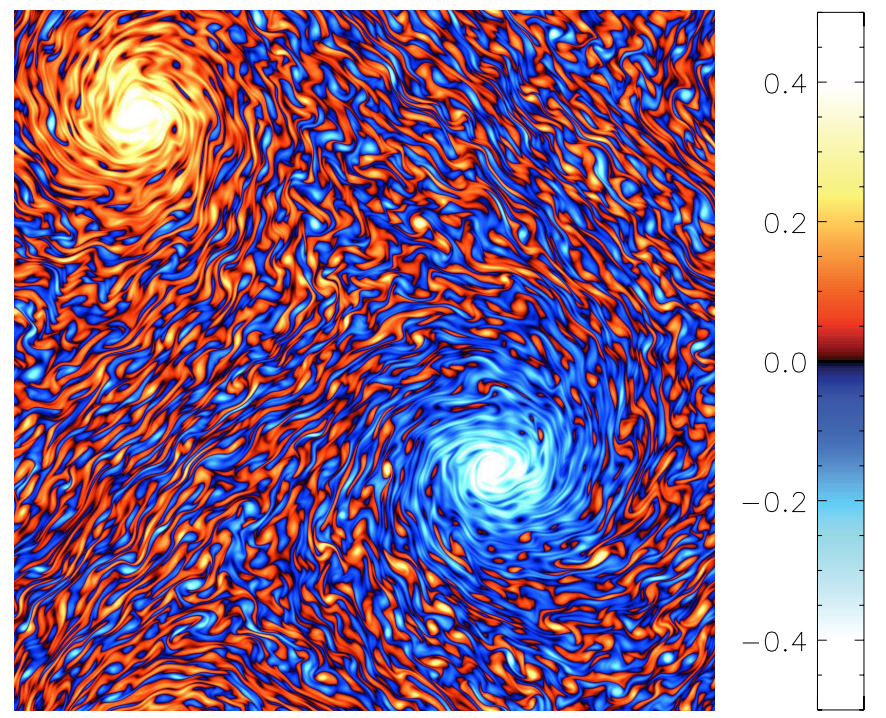

FIG. 1: (Color online) Pseudocolor plot of vorticity showing Bose condensation. Red (the top-left vortex) and blue (the bottom-right vortex) represent positive and negative vorticity in physical space, respectively. The color scale is shown in the color bar on the right, which is chosen to make the fluctuation visible. The vorticity is normalized so that $\max |\omega|=1$. 


\begin{tabular}{|c|c|c|c|c|c|c|c|c|c|c|}
\hline \multirow[b]{2}{*}{ Name } & \multicolumn{2}{|c|}{ Inputs } & \multicolumn{3}{|c|}{ Predictions } & \multicolumn{4}{|c|}{ Results } & \multirow[b]{2}{*}{ Notes } \\
\hline & $\nu$ & $k_{\mathrm{i}}$ & $k_{\mathrm{d}}$ & $\tau_{\bar{E}}$ & $E_{\infty}$ & Resolution & $\tilde{\tau}_{\bar{E}}$ & $\tilde{E}_{\infty}$ & $\tilde{E}_{\infty} / E_{\infty}$ & \\
\hline a16 & $10^{-3}$ & 16 & 80 & 500 & 405 & $512^{2}$ & 483 & 323 & 0.80 & \\
\hline a24 & $10^{-3}$ & 24 & 91 & 500 & 356 & $512^{2}$ & 520 & 281 & 0.79 & \\
\hline a32 & $10^{-3}$ & 32 & 100 & 500 & 308 & $512^{2}$ & 582 & 234 & 0.76 & \\
\hline $\mathrm{b} 16^{\mathrm{f}}$ & $5 \times 10^{-4}$ & 16 & 113 & 1000 & 891 & $512^{2}$ & - & - & - & single-precision, crashed \\
\hline $\mathrm{b} 16$ & $5 \times 10^{-4}$ & 16 & 113 & 1000 & 891 & $512^{2}$ & 922 & 696 & 0.78 & \\
\hline b24 & $5 \times 10^{-4}$ & 24 & 129 & 1000 & 832 & $512^{2}$ & 947 & 643 & 0.77 & \\
\hline b32 & $5 \times 10^{-4}$ & 32 & 142 & 1000 & 772 & $512^{2}$ & 970 & 580 & 0.75 & \\
\hline c16 & $2 \times 10^{-4}$ & 16 & 178 & 2500 & 2373 & $1024^{2}$ & 2223 & 1818 & 0.76 & \\
\hline c32 & $2 \times 10^{-4}$ & 32 & 224 & 2500 & 2226 & $1024^{2}$ & 2287 & 1714 & 0.77 & \\
\hline c64 & $2 \times 10^{-4}$ & 64 & 283 & 2500 & 1928 & $1024^{2}$ & 2409 & 1385 & 0.72 & \\
\hline $\mathrm{c} 64^{\mathrm{b}}$ & $2 \times 10^{-4}$ & 64 & 283 & 2500 & 1928 & $512^{2}$ & 1985 & 791 & 0.41 & converge to wrong answer \\
\hline d16 & $10^{-4}$ & 16 & 252 & 5000 & 4859 & $1024^{2}$ & 4145 & 3581 & 0.74 & \\
\hline d32 & $10^{-4}$ & 32 & 317 & 5000 & 4691 & $1024^{2}$ & 4006 & 3349 & 0.72 & \\
\hline path $^{i}$ & $5 \times 10^{-4}$ & 32 & 142 & 1000 & 308 & $512^{2}$ & - & 580 & 0.75 & restart from saturated b32 \\
\hline
\end{tabular}

TABLE I: List of simulations: The first 13 simulations are used to verify our three-scale model (16). The last row describes 16 restart-runs which are initially in saturated states. They are used to verify the properties of $\Delta r^{2}$. The details of the simulations are described in Sec. II.

\section{NUMERICAL SIMULATIONS}

Let $\psi$ be the 2D (scalar) stream function. The velocity is then $\boldsymbol{u}=\left(\partial_{y} \psi,-\partial_{x} \psi\right)$ and the $z$ component of the vorticity is $\omega=-\nabla^{2} \psi$. We solve the $2 \mathrm{D}$ incompressible Navier-Stokes equations in the vorticity-stream-function formulation, that is,

$$
\partial_{t} \omega-J(\psi, \omega)=\nu \nabla^{2} \omega+g,
$$

where the Jacobian determinant is given by

$$
J(\psi, \omega)=\left(\partial_{x} \psi\right)\left(\partial_{y} \omega\right)-\left(\partial_{x} \omega\right)\left(\partial_{y} \psi\right) .
$$

We use periodic boundary conditions with $L=2 \pi$ so $k_{1}=1$, and denote the Fourier transform of $\omega$ by $\omega_{\boldsymbol{k}}$.

In Eq. (1), $g$ is the $z$ component of the curl of an external force. Using $\langle\cdot\rangle$ to denote ensemble averages, the Fourier transform of the force, $g_{\boldsymbol{k}}$, is taken to be random and white-in-time with zero means and variance

$$
\left\langle g_{\boldsymbol{k}}^{*}(s) \cdot g_{\boldsymbol{k}}(t)\right\rangle=f_{\mathrm{i}}^{2} k_{\mathrm{i}}^{2} \delta(t-s) .
$$

It is achieved by choosing a random phase for $g_{\boldsymbol{k}_{\mathrm{i}}}$ at each time step. In the above expression, $f_{\mathrm{i}}$ is the forcing amplitude and $k_{\mathrm{i}}$ is the forcing wave number. To ensure isotropy, we select $\boldsymbol{k}$ randomly in a shell with radius $k_{\mathrm{i}}$ and then round it off to the nearest grid point in Fourier space. The effective width of the shell is approximately $k_{1}$. For this force, the average rates of energy and enstrophy inputs are respectively $f_{\mathrm{i}}^{2}$ and $f_{\mathrm{i}}^{2} k_{\mathrm{i}}^{2}$.

We use a spectral Galerkin scheme in space and a lowstorage third-order Runge-Kutta/Crank-Nicolson time stepping scheme [13]. The time steps $\Delta t$ are chosen with a Courant number of 0.5. The non-linear term is treated explicitly and the diffusion term is treated implicitly. The stochastic forcing is integrated by the Euler-Maruyama method [14]. We use $N \times N$ grid points with the Galerkin cutoff at $k_{\mathrm{G}} \equiv\lfloor(N-1) / 3\rfloor+0.99$, where $\lfloor\cdot\rfloor$ denotes the floor function. The value 0.99 is chosen such that the comparison $k^{2} \leq k_{\mathrm{G}}^{2}$ is accurate enough even in single precision with $N \sim 2048$, although almost all simulations in this paper are done with double precision.

Our code is implemented in CUDA $\mathrm{C}$ and runs on graphics processing units (GPUs), which are massively parallel "stream" processors. With an nVidia Tesla C2050 graphic card, our code is over an order of magnitude faster than codes running on a single CPU core. Because of communication overhead, our code outperforms distributed-memory parallel codes running on 32 cores. This speed-up allows us to integrate the problem over very long code time and study the saturation of the condensate vortices. We have released our Spectral Galerkin $2 \mathrm{D}$ code (sg2) as an open-source project under the GNU General Public License version 3. The project is hosted by the Google Code http://sg2.googlecode.com/.

\section{RESULTS}

We have performed a series of simulations as shown in Table I with different sets of input parameters $\nu$ and $k_{\mathrm{i}}$. In all of them, the initial conditions are $\omega_{\boldsymbol{k}}(t=0)=0$. We set the forcing amplitude at $f_{\mathrm{i}}=1$ and choose the forcing wave number $k_{\mathrm{i}}$ so that $k_{1} \ll k_{\mathrm{i}} \ll k_{\mathrm{d}}$. The dissipation wave number $k_{\mathrm{d}}$ is given by

$$
k_{\mathrm{d}} \equiv \eta^{1 / 6} \nu^{-1 / 2} \text {, }
$$

where $\eta$ is the forward enstrophy flux. Further details of the runs are given in Table I. 


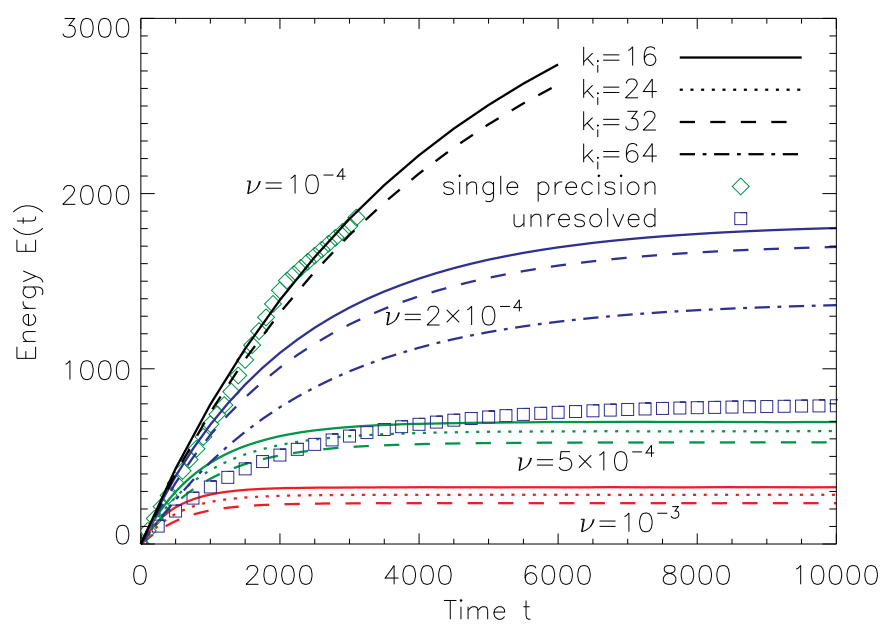

FIG. 2: (Color online) Unnormalized energy evolution in simulations a16 - d32. The thick red (lowermost), green, blue, and black (uppermost) curves are for different viscosity $\nu$ (see labels); while solid, dashed, and dotted styles denote different forcing wave number $k_{\mathrm{i}}$ (see legend). The green diamonds and blue squares are for simulations $\mathrm{b} 16^{\mathrm{f}}$ and $\mathrm{c} 64^{\mathrm{b}}$, respectively. They correspond to single precision and under-resolved simulations.

In our simulations, energy inverse cascades to small Fourier modes till $k_{1}$ and forms a condensate at this mode. We plot the total energy $E(t)$ for simulations a16 - d32 in Fig. 2. The different colors and line styles represent different choices of viscosity $\nu$ (see labels) and forcing scale $k_{\mathrm{i}}$ (see legend). The blue squares and green diamonds correspond to a single precision $\left(\mathrm{b} 16^{\mathrm{f}}\right)$ and an under-resolved $\left(c 64^{b}\right)$ simulations, which we will comment in Sec. IV. For intermediate time, the growth of energy of this condensate is consistent with earlier results [7]. Nevertheless, the asymptotic $E(t)$ becomes independent of time in all the cases. This saturation happens at a time scale determined by the viscous time at the largest length scale (i.e., $\tau_{\nu}=1 / \nu k_{1}^{2}$ ). This requires very long integration times and hence has not been explored by earlier simulations. We are able to reach such late times by virtue of using a GPU code.

At later times, most of the total energy comes from the energy of the condensate. Further understanding of the growth and saturation of the condensate can be obtained by studying the energy spectrum. We compute the shell-integrated energy spectrum $E_{k}$ in simulation c32 at $t=1000$ using a fixed bin size $\Delta k=k_{1}$. The result is plotted as the thick solid curve in Fig. 3. The condensate is well developed but not fully saturated. The dashed and dotted lines in the figure are proportional to $k^{-5 / 3}$ and $k^{-3}$, respectively. Although the inertial ranges are narrow, the inverse energy cascade and forward enstrophy cascade are consistent with spectral slope $-5 / 3$ and -3 . However, the condensate strongly deviates the inverse cascade spectrum at large scale. This additional feature motivates us to introduce a simple three-scale model.

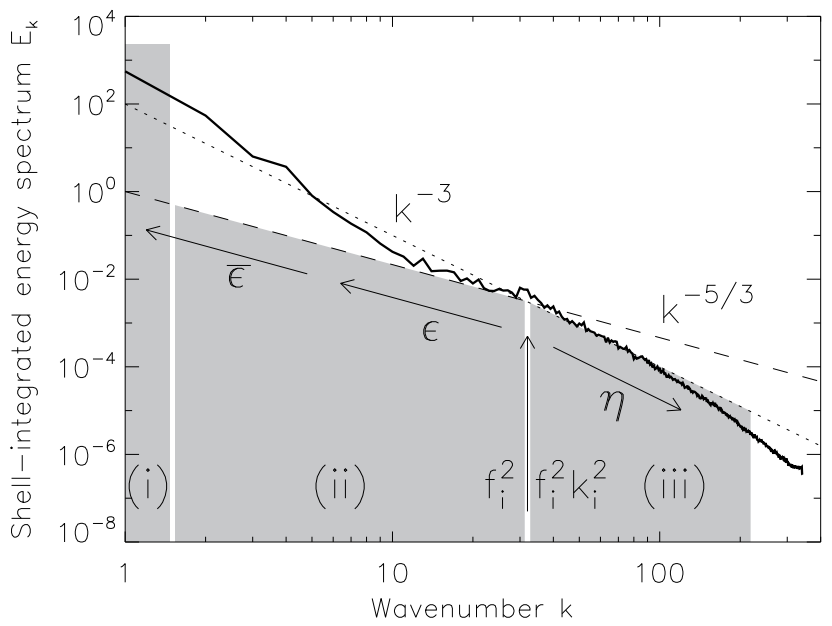

FIG. 3: Energy spectrum in simulation c32. The thick solid curve is the energy spectrum $E_{k}$ computed at $t=1000$ with bin size $\Delta k=k_{1}$. The dashed line shows $k^{-5 / 3}$; while the dotted line is proportional to $k^{-3}$. The gray areas, which represent our three-scale model, are drawn in proper scales. The condensate at zone (i) contains a large amount of energy. The broken power law in zones (ii) and (iii) have slopes $-5 / 3$ and -3 , respectively. See Sec. III A for details of the model.

\section{A. Energy and enstrophy evolution}

Given the energy spectrum $E_{k}$, the total energy, enstrophy, and palinstrophy can be computed by the onedimensional integrals $E=\int E_{k} d k, Z=\int k^{2} E_{k} d k$, and $P=\int k^{4} E_{k} d k$, respectively. Together with the average energy and enstrophy input, the evolution of these quantities is governed by the following equations

$$
\begin{aligned}
& \partial_{t} E=-2 \nu Z+f_{\mathrm{i}}^{2}, \\
& \partial_{t} Z=-2 \nu P+f_{\mathrm{i}}^{2} k_{\mathrm{i}}^{2} .
\end{aligned}
$$

Our three-scale model is constructed in the following fashion. We refer to the corresponding wave number ranges as zones (i) through (iii) as shown in Fig. 3. (i) At the wave number of the box $k_{1}$, we have the condensate modes, which contain most of the energy at saturation. We use the shorthand $\bar{E}(t)$ to denote the timedependent energy at this Fourier mode. (ii) In the range $k_{1}<k \leq k_{\mathrm{i}}$, the energy inverse cascades from the forcing wave number $k_{\mathrm{i}}$ and forms an $E_{k} \sim k^{-5 / 3}$ spectrum. Note that the enstrophy spectrum $Z_{k}=k^{2} E_{k}$ peaks at the forcing wave number $k_{\mathrm{i}}$. (iii) The forward enstrophy cascade in the small scales gives the spectrum $E_{k} \sim k^{-3}$ with a sharp cutoff at $|\boldsymbol{k}|=k_{\mathrm{d}}$. It is necessary to assume this cutoff to prevent the ultraviolet divergence of the total enstrophy and palinstrophy, although not total energy.

Let us now denote by $\bar{E}$ the energy in the $|\boldsymbol{k}|=k_{1}$ mode (i.e., the condensate) and by $E^{\prime}$ the energy of the rest of the system (i.e., $E=\bar{E}+E^{\prime}$ ). Following standard mean-field theory, we call them, respectively, the mean 
and fluctuating parts, even though $E^{\prime}$ contributes to the coherent structure as we will show in Sec. III C. Similar notations are applied to the enstrophy and palinstrophy too. We can then split the energy equation (5) into two parts, namely,

$$
\begin{aligned}
\partial_{t} \bar{E} & =-2 \nu k_{1}^{2} \bar{E}+\bar{\epsilon} \\
\partial_{t} E^{\prime} & =-2 \nu Z^{\prime}+f_{\mathrm{i}}^{2}-\bar{\epsilon}
\end{aligned}
$$

where $\bar{\epsilon}$ is the inverse energy transfer rate to the condensate. Similarly, with the help of Eq. (7), we can rewrite Eq. (6) as

$$
\partial_{t} Z^{\prime}=-2 \nu P^{\prime}+f_{\mathrm{i}}^{2} k_{\mathrm{i}}^{2}-k_{1}^{2} \bar{\epsilon}
$$

The last term $k_{1}^{2} \bar{\epsilon}$ describes the enstrophy transfer rate to the condensate. Its existence implies that, although most of the enstrophy is transferred toward smaller scales, there is a small leakage towards the largest scale.

Equations (7) through (9) are exact but not closed. Hence, it is not yet possible to solve them simultaneously. We parametrize the fluctuating palinstrophy and enstrophy by $\gamma$ and $\Gamma$ such that

$$
\begin{aligned}
P^{\prime} & \equiv P-k_{1}^{4} \bar{E}=\gamma k_{\mathrm{d}}^{2} Z^{\prime}, \\
Z^{\prime} & \equiv Z-k_{1}^{2} \bar{E}=\Gamma k_{\mathrm{i}}^{2} E^{\prime} .
\end{aligned}
$$

Substituting the above equations into Eqs. (8) and (9) we obtain two simultaneous equations. We then eliminate $\bar{\epsilon}$ between them to obtain a dynamical equation for $Z^{\prime}$, which can be solved to obtain

$$
Z^{\prime}(t)=Z_{\infty}^{\prime}\left[1-e^{-\left(t-t_{0}\right) / \tau_{Z^{\prime}}}\right]
$$

where we have used the initial condition $Z^{\prime}\left(t_{0}\right)=0$ and

$$
Z_{\infty}^{\prime} \equiv \frac{f_{\mathrm{i}}^{2}}{2 \nu} \frac{k_{\mathrm{i}}^{2}-k_{1}^{2}}{\gamma k_{\mathrm{d}}^{2}-k_{1}^{2}}
$$

with characteristic time scale

$$
\tau_{Z^{\prime}} \equiv \frac{1}{2 \nu \Gamma k_{\mathrm{i}}^{2}} \frac{\Gamma k_{\mathrm{i}}^{2}-k_{1}^{2}}{\gamma k_{\mathrm{d}}^{2}-k_{1}^{2}} .
$$

Now note that the enstrophy saturation time scale $\tau_{Z^{\prime}} \approx 1 / 2 \nu \gamma k_{\mathrm{d}}^{2}$ is substantially faster than the energy saturation time scale, which is of the order of $\nu k_{1}^{2}$. Hence, for simplicity, we can replace $\bar{\epsilon}$ by its value at late time in Eq. (7), which can be obtained by replacing $Z^{\prime}$ by $Z_{\infty}^{\prime}$ in Eq. (8),

$$
\bar{\epsilon}_{\infty} \equiv \lim _{t \rightarrow \infty} \bar{\epsilon}(t)=f_{\mathrm{i}}^{2}-2 \nu Z_{\infty}^{\prime}=f_{\mathrm{i}}^{2} \frac{\gamma k_{\mathrm{d}}^{2}-k_{\mathrm{i}}^{2}}{\gamma k_{\mathrm{d}}^{2}-k_{1}^{2}}
$$

Substituting this back into Eq. (7) and solving for $\bar{E}$, we obtain

$$
\bar{E}(t)=\bar{E}_{\infty}\left[1-e^{-\left(t-t_{0}\right) / \tau_{\bar{E}}}\right]
$$

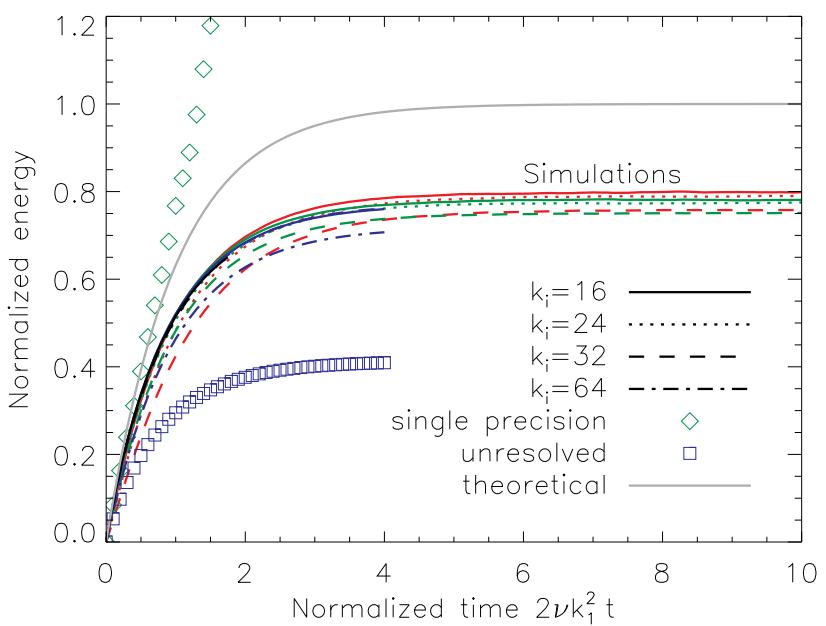

FIG. 4: (Color online) Normalized energy evolution in simulations a16 - d32. The thick red, green, blue, and black curves (all under the "Simulations" label) are for different viscosity (see also labels in Fig. 2); while solid, dashed, and dotted styles denote different forcing wave number $k_{\mathrm{i}}$ (see legend). We normalize the energy and time using the results from our three-scale model. Almost all the curves collapse onto each other. The gray solid curve, green diamonds, and blue squares are the theoretical prediction, $\mathrm{b} 16^{\mathrm{f}}$, and $\mathrm{c} 64^{\mathrm{b}}$, respectively.

where we have used the initial condition $\bar{E}\left(t_{0}\right)=0$. This solution saturates to

$$
\bar{E}_{\infty} \equiv \frac{f_{\mathrm{i}}^{2}}{2 \nu k_{1}^{2}} \frac{\gamma k_{\mathrm{d}}^{2}-k_{\mathrm{i}}^{2}}{\gamma k_{\mathrm{d}}^{2}-k_{1}^{2}}
$$

with a characteristic time scale

$$
\tau_{\bar{E}} \equiv 1 / 2 \nu k_{1}^{2}
$$

In the saturation level (17), the first part $f_{\mathrm{i}}^{2} / 2 \nu k_{1}^{2}$ can be easily derived by dimensional arguments; while the correction factor $\left(\gamma k_{\mathrm{d}}^{2}-k_{\mathrm{i}}^{2}\right) /\left(\gamma k_{\mathrm{d}}^{2}-k_{1}^{2}\right)<1$ comes from our three-scale model. This result is consistent with the energy bounds derivation by Eyink [10].

To obtain the values of $\bar{E}_{\infty}$, we need to solve for $k_{\mathrm{d}}$ and $\gamma$. For the former, we combine definition (4) and the forward enstrophy flux in Eq. (9) to obtain

$$
k_{\mathrm{d}}=f_{\mathrm{i}}^{1 / 3}\left(k_{\mathrm{i}}^{2}-k_{1}^{2}\right)^{1 / 6} \nu^{-1 / 2} .
$$

For the latter, we assume that in the inverse cascade $E_{k} \sim k^{-5 / 3}$, and in the direct cascade $E_{k} \sim k^{-3}$. These two power laws must match at $k=k_{\mathrm{i}}$. Using these, we can compute the dimensionless number

$$
\gamma \approx \frac{2}{3+4 \ln \left(k_{\mathrm{d}} / k_{\mathrm{i}}\right)} \lesssim 1
$$

With this, the model no longer has any free parameter. In Fig. 4, we re-plot $E(t)$ with the vertical axis normalized by $\bar{E}_{\infty}$ and also scale the time axis by $\tau_{\bar{E}}$. This collapses all the different time traces, thus providing support to our three-scale model. Note that the non-trivial 
correction factor given in Eq. (17) is necessary to obtain this collapse. Note also that, the expressions for $\bar{E}_{\infty}$ and $Z_{\infty}^{\prime}$ (but not the time scales) can be derived directly by demanding that we reach a stationary state at late times. Imposing the conditions $\partial_{t} E=\partial_{t} Z=0$ to Eqs. (5) and (6), Eqs. (17) and (13) follow immediately.

Although all the results from different simulations collapse onto each other, this collapsed curve lies systematically below the analytical solution shown as light gray curve in Fig. 4. To estimate this discrepancy, we fit the numerical curves by the form of Eq. (16) to find numerical values of the total energy and the saturation time scale. These values are compared with their theoretical prediction in Table I. The time scales agree to a good accuracy. The ratios between the theoretical $E_{\infty}$ and the fitted $\tilde{E}_{\infty}$ are listed in the tenth column of Table I. The average of these ratios (not including $\mathrm{b} 16^{\mathrm{f}}, \mathrm{c} 64^{\mathrm{b}}$, and path $\left.^{\mathrm{i}}\right)$ is $\zeta \equiv\left\langle\tilde{E}_{\infty} / E_{\infty}\right\rangle \approx 0.76$. We speculate that this systematic difference exists because we have ignored the feedback of the condensate to the spectrum in our threescale model. We will discuss this speculation with more details in Sec. IV.

Comments on the remaining run in Table I are now in order. The blue squares are for the $c 64^{\mathrm{b}}$ run. Although they look perfectly reasonable, the resolution of the simulation, $512^{2}$, which gives $k_{\mathrm{G}} \approx 170$, is too low to resolve the actual enstrophy dissipation wave number $k_{\mathrm{d}}=283$. Because spectral Galerkin methods have very little numerical dissipation, the forward cascaded enstrophy cannot be removed, which artificially increases $Z_{\infty}^{\prime}$ and decrease $\bar{\epsilon}_{\infty}$. Hence, the steady state energy $E_{\infty}$ converges to an (incorrect) lower value. In the language of numerical analysis, this is an inconsistent numerical solution.

The green diamonds in Fig. 4 are for the $\mathrm{b} 16^{\mathrm{f}}$ run, which uses single-precision floating-point numbers to represent $\omega_{\boldsymbol{k}}$ instead of double precision. The numerical solution behaves properly at the beginning, but starts to diverge from the true solution around $t=1000=\tau_{\bar{E}}$. This is not surprising because the relative round-off error of single-precision numbers is about $\sim 10^{-7}$. A random walk of round-off error in $\omega_{\boldsymbol{k}}$ leads to a linear grow of error in the energy. Indeed, there are roughly $10^{7}$ steps by the time we reach $t=\tau_{\bar{E}}$, which gives the order of unit error seen in the figure. To date, a significant fraction of scientific computing in GPUs are done with single precision. This turns out to be insufficient for our purpose.

\section{B. Movement of Condensate Vortices}

After studying the saturation level and time scale, we focus on the movement of the condensate vortices at late times. The last row in Table I represents 16 different simulations path ${ }^{85}-$ path $^{100}$. The superscripts in the names have the following meaning. For path ${ }^{i}$, we pick the i-th output file of b32 and restart the simulation each time with a different realization of the random force. In other words, for the i-th run the random number generators are started with seed $i$. We then evolve the solutions for another $t=10 \tau_{\bar{E}}$. Because $\mathrm{b} 32$ has reached a saturated state long before the 85-th output, the energy levels remain almost constant in all these simulations. The result is an ensemble of 16 saturated solutions [15].

The positions of the condensate vortices are simply given by the phases of the $k_{1}$ modes. We unfold the trajectories from the computational domain $[0,2 \pi)^{2}$ into $\mathbb{R}^{2}$ and shift their starting points to the origin. We label respectively the $x$ - and $y$-displacements, respectively, by $\Delta x$ and $\Delta y$ and plot such a trajectory (for path ${ }^{100}$ ) in Fig. 5. The small gray box near the origin shows the size of the original domain $[0,2 \pi)^{2}$. With the unfolded trajectories, we compute the displacement square $\Delta r^{2}=$ $\Delta x^{2}+\Delta y^{2}$ for all 16 path ${ }^{i}$ runs. For each simulation, we plot a gray curve in Fig. 6. The thick solid curve shows their (ensemble) average. In the following, we describe a way to model the motion of this condensate.

We define the "mean" vorticity by

$$
\bar{\omega}(\boldsymbol{x}) \equiv \sum_{|\boldsymbol{k}|=k_{1}} \omega_{\boldsymbol{k}} e^{i \boldsymbol{k} \cdot \boldsymbol{x}}
$$

so the fluctuating vorticity is $\omega^{\prime}=\omega-\bar{\omega}$. Averaging the Navier-Stokes equation, we obtain

$$
\partial_{t} \bar{\omega}+\nabla \cdot(\overline{\boldsymbol{u}} \bar{\omega})=-\nabla \cdot \overline{\mathcal{F}}+\nu \nabla^{2} \bar{\omega} .
$$

In the above equation, $\overline{\mathcal{F}} \equiv \overline{\boldsymbol{u} \omega}-\overline{\boldsymbol{u}} \bar{\omega}$ is the spacedependent mean vorticity flux. Note that the forcing $g$ is at small wave numbers $k_{\mathrm{i}} \gg k_{1}$, so its mean vanishes. It is straightforward to show that the non-linear term $\boldsymbol{\nabla} \cdot(\overline{\boldsymbol{u}} \bar{\omega})$ vanishes identically. Both the creation and the movement of the condensate, therefore, must be due to the flux $\overline{\mathcal{F}}$.

We now model this mean vorticity flux using the usual technique of mean-field theory

$$
\overline{\mathcal{F}}_{i}=\alpha_{i} \bar{\omega}+\beta_{i j} \partial_{j} \bar{\omega} .
$$

The transport coefficients $\alpha_{i}$ is usually referred to as the kinetic anisotropic alpha effect [16-18], and $\beta_{i j}$ is a negative eddy diffusivity tensor. Both $\alpha_{i}$ and $\beta_{i j}$ are constants in space because we consider only the $k_{1}$ modes. The mean-field equation then becomes

$$
\left(\partial_{t}+\alpha_{i} \partial_{i}\right) \bar{\omega}=\left(\nu \delta_{i j}-\beta_{i j}\right) \partial_{i} \partial_{j} \bar{\omega} .
$$

The coefficient $\alpha_{i}$ cannot change the amplitude of the condensate. It can be thought of as the Lagrangian velocity of the pair of condensate vortices. The inverse energy cascade, therefore, must be described by anti-diffusion.

Expanding $\boldsymbol{\nabla} \cdot \overline{\mathcal{F}}$, there are only two independent Fourier coefficients, $\overline{\mathcal{F}}_{x, k_{1} \hat{\boldsymbol{x}}}$ and $\overline{\mathcal{F}}_{y, k_{1} \hat{\boldsymbol{y}}}$, that enter the mean-field equation (the first subscript denotes component, the second one denotes wave vector). Comparing 


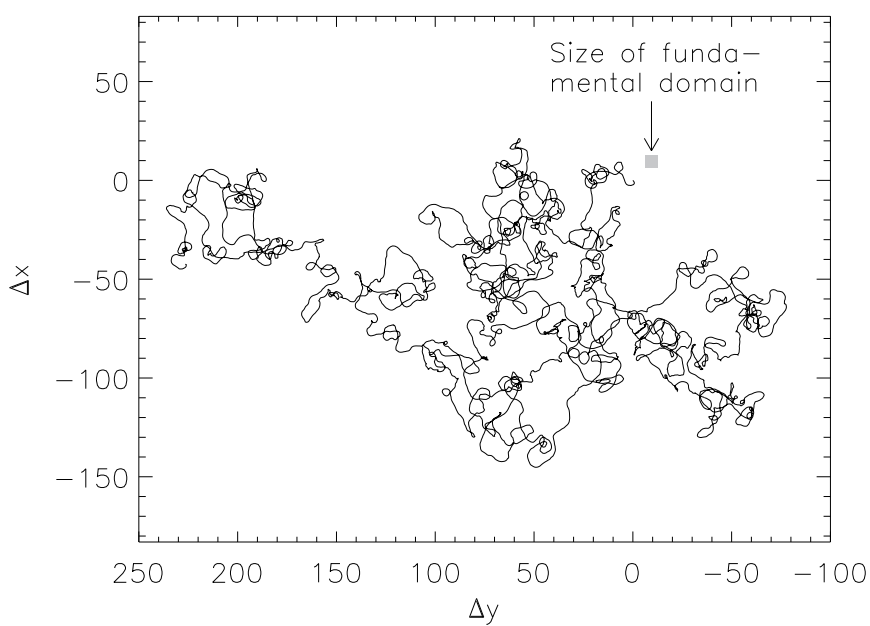

FIG. 5: Unfolded trajectory of the positive condensate vortex in simulation path ${ }^{100}$. The computational domain is $[0,2 \pi)^{2}$ (see gray box near the origin). We first unfold the trajectory into $\mathbb{R}^{2}$ and then shift the initial position to the origin.

to our parametrization (23),

$$
\begin{aligned}
\alpha_{x} & =\operatorname{Re} \frac{\overline{\mathcal{F}}_{x, k_{1} \hat{\boldsymbol{x}}}}{\omega_{k_{1} \hat{\boldsymbol{x}}}}, & \alpha_{y} & =\operatorname{Re} \frac{\overline{\mathcal{F}}_{y, k_{1} \hat{\boldsymbol{y}}}}{\omega_{k_{1} \hat{\boldsymbol{y}}}} ; \\
\beta_{x x} & =\operatorname{Im} \frac{\overline{\mathcal{F}}_{x, k_{1} \hat{\boldsymbol{x}}}}{k_{1} \omega_{k_{1} \hat{\boldsymbol{x}}}}, & \beta_{y y} & =\operatorname{Im} \frac{\overline{\mathcal{F}}_{y, k_{1} \hat{\boldsymbol{y}}}}{k_{1} \omega_{k_{1} \hat{\boldsymbol{y}}}} .
\end{aligned}
$$

It is clear that $\left\langle\beta_{x x}\right\rangle=\left\langle\beta_{y y}\right\rangle=\nu$ at saturation.

The value of $\boldsymbol{\alpha}$, however, is much more difficult to obtain because there is no constraint by conservation laws. We can only perform a rough estimate: The flux $\overline{\mathcal{F}}$ is a convolution in Fourier space. Given that $k_{1}^{2} \bar{E}_{\infty} / Z_{\infty}^{\prime} \gg 1$, the condensate-fluctuation interaction dominates, so

$$
\overline{\mathcal{F}} \sim \boldsymbol{u}_{\sqrt{2} k_{1}} \bar{\omega}
$$

where $\boldsymbol{u}_{\sqrt{2} k_{1}}$ represents the typical value of a band-pass filtered velocity. Comparing the estimate with Eq. (25) and employing the results from our three-scale model, we obtain

$$
\left\langle\alpha^{2}\right\rangle \sim k_{1} E_{\sqrt{2} k_{1}} \approx \frac{f_{\mathrm{i}}^{2}}{2 \nu k_{\mathrm{d}}^{2}}\left(\frac{k_{\mathrm{i}}}{k_{1}}\right)^{2 / 3}=\frac{f_{\mathrm{i}}^{4 / 3}}{2 k_{1}^{2 / 3}} .
$$

Note that the same argument leads to $\left\langle\beta^{2}\right\rangle \sim k_{1}^{-2}\left\langle\alpha^{2}\right\rangle$. Fortunately, it does not contradict $\langle\beta\rangle=\nu$.

Let us now take $\boldsymbol{\alpha}$ to be statistically independent of $\bar{\omega}$. Equation (24) then becomes the equation of a passive scalar advected by a random velocity $\boldsymbol{\alpha}$. As $\boldsymbol{\alpha}$ has a stationary variance (28), its simplest model would be an Ornstein-Uhlenbeck process [19-21]

$$
\partial_{t} \boldsymbol{\alpha}=-\boldsymbol{\alpha} / \tau_{\alpha}+\boldsymbol{\phi}
$$

In the above phenomenological equation, $1 / \tau_{\alpha}$ is an effective drag coefficient and $\phi$ is an effective stochastic

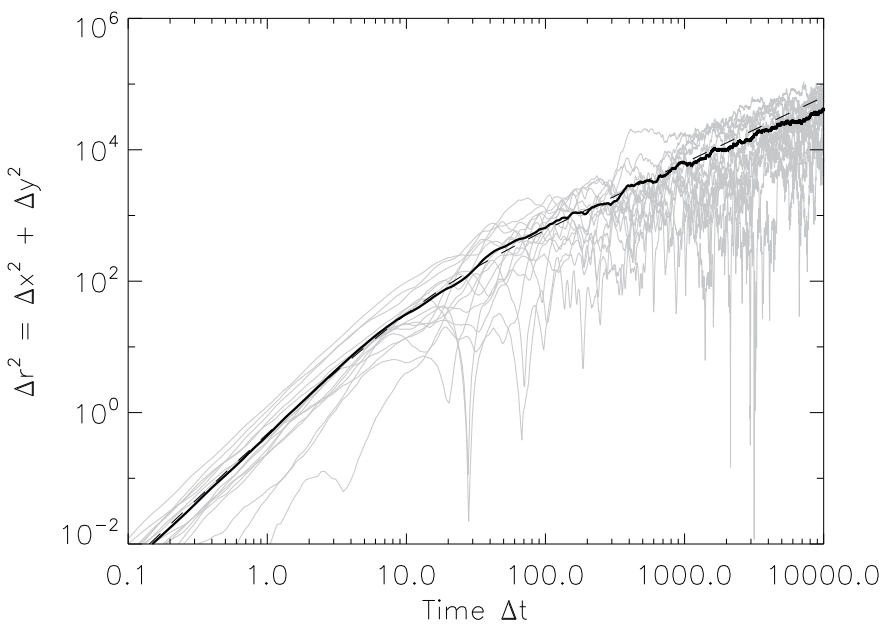

FIG. 6: The gray curves are the displacement square $\Delta r^{2}$ for all 16 path $^{i}$ runs. The thick solid curve shows their average. The dashed curve, which is indistinguishable from the solid curve, is the solution (35) with parameter $\xi=0.1$.

forcing. Because $\boldsymbol{\alpha}$ is at a scale close to the condensate, we model the effective forcing $\phi$ by Gaussian white noise with zero mean and variance equal to $\xi \bar{\epsilon}$. Here $\xi$ is a tunable parameter and $\bar{\epsilon}$ is the mean energy input to the condensate. Standard Itō calculus gives

$$
\begin{aligned}
& \langle\boldsymbol{\alpha}(t)\rangle=\boldsymbol{\alpha}(0) e^{-t / \tau_{\alpha}}, \\
& \langle\boldsymbol{\alpha}(s) \cdot \boldsymbol{\alpha}(t)\rangle=\alpha(0)^{2} e^{-(t+s) / \tau_{\alpha}} \\
& \quad+\xi f_{\mathrm{i}}^{2} \frac{\gamma k_{\mathrm{d}}^{2}-k_{\mathrm{i}}^{2}}{\gamma k_{\mathrm{d}}^{2}-k_{1}^{2}} \tau_{\alpha}\left[e^{-(t-s) / \tau_{\alpha}}-e^{-(t+s) / \tau_{\alpha}}\right],
\end{aligned}
$$

where the second equation is only valid for $s<t$.

By requiring $\left\langle\alpha(t)^{2}\right\rangle=\left\langle\alpha^{2}\right\rangle$ for arbitrary large $t$, we can solve for the time scale

$$
\tau_{\alpha}=\frac{1}{2 \xi f_{\mathrm{i}}^{2 / 3} k_{1}^{2 / 3}} \frac{\gamma k_{\mathrm{d}}^{2}-k_{1}^{2}}{\gamma k_{\mathrm{d}}^{2}-k_{\mathrm{i}}^{2}}
$$

Furthermore, starting with an expected initial condition $\alpha(0)^{2}=\left\langle\alpha^{2}\right\rangle$ allows us to simplify the correlation to

$$
\langle\boldsymbol{\alpha}(s) \cdot \boldsymbol{\alpha}(t)\rangle=\left\langle\alpha^{2}\right\rangle e^{-(t-s) / \tau_{\alpha}} .
$$

The displacement of the condensate can now be solved by using the simple equation

$$
\partial_{t} \boldsymbol{r}=\boldsymbol{\alpha} .
$$

The motion of an inertial particle in a fluid with Stokes drag is described by the same pair of equations [Eqs. (29) and (34)]. The role of effective velocity is played by $\boldsymbol{\alpha}$ and the effective Stokes time is given by $\tau_{\alpha}$.

Let $\Delta \boldsymbol{r}(t) \equiv \boldsymbol{r}(t)-\boldsymbol{r}(0)$ be the displacement from the initial position, we have

$$
\begin{aligned}
\left\langle\Delta r(t)^{2}\right\rangle & =2 \int_{0}^{t} d t^{\prime} \int_{0}^{t^{\prime}} d s^{\prime}\left\langle\boldsymbol{\alpha}\left(s^{\prime}\right) \cdot \boldsymbol{\alpha}\left(t^{\prime}\right)\right\rangle \\
& =2\left\langle\alpha^{2}\right\rangle \tau_{\alpha}\left[t+\tau_{\alpha}\left(e^{-t / \tau_{\alpha}}-1\right)\right] .
\end{aligned}
$$




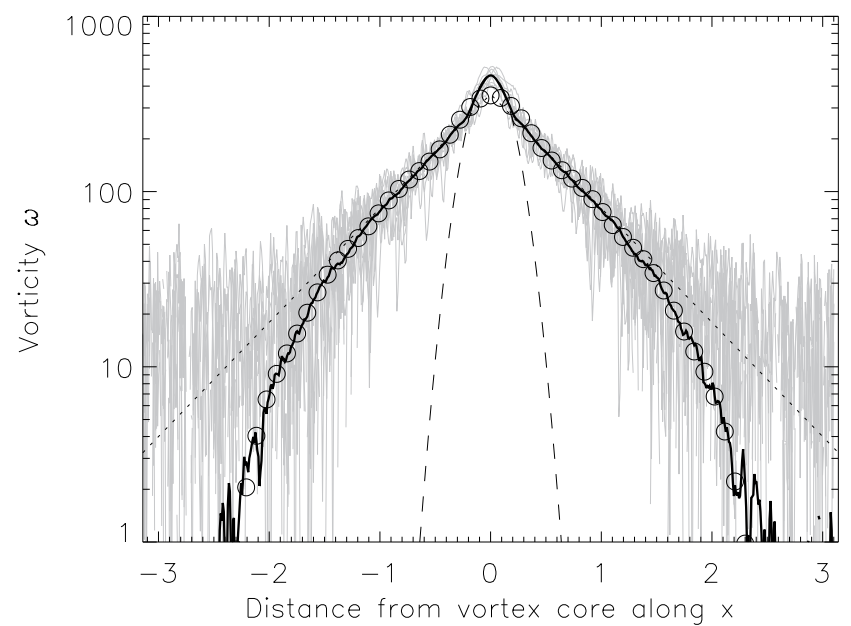

FIG. 7: We apply coordinate transformations to shift the center of the positive condensate vortex to the origin in simulation c32. The noisy gray curves show the vorticity at several different snapshots along the $x$-axis (with $y=0$ ). The black solid curve is an average over 1000 such profiles. It consists of a sharp Gaussian core (dashed curve) and exponential wings (dotted curve), although for $|x| \gtrsim \pi / 2$ it falls off superexponentially. The open circles show the reconstruction from the Fourier coefficients listed in Table II.

Its asymptotic behavior is

$$
\left\langle\Delta r(t)^{2}\right\rangle \approx\left\{\begin{array}{cc}
\left\langle\alpha^{2}\right\rangle t^{2}, & t<\tau_{\alpha} \\
2\left\langle\alpha^{2}\right\rangle \tau_{\alpha} t, & t>\tau_{\alpha}
\end{array}\right.
$$

The early time behavior is completely determined by $\left\langle\alpha^{2}\right\rangle$, while the free parameter $\xi$ controls only the Brownian motion of the condensate at late time.

The dashed curve in Fig. 6 is Eq. (35) with a parameter $\xi=0.1$, which corresponds to $\tau_{\alpha}=6.384$. It is practically indistinguishable from the thick curve, except at very late times $(\Delta t>3000)$. Note that at short times the condensate movement is independent of $\xi$. Hence the agreement between the thick solid and dashed curves for $\Delta t<\tau_{\alpha}$ provides support to our phenomenological model. Although we are not able to derive $\xi$, the late time agreement suggests that the condensate motion is Brownian for $\Delta t>\tau_{\alpha}$.

\section{Shape of Coherent Vortices}

We can invert the procedure of computing the vortex trajectory to hold fixed the positive condensate vortex at the origin of the computational domain. In Fig. 7, the noisy gray curves show ten different profiles of the condensate along the $x$ axis (with $y=0$ ). Performing an average over 1000 such profiles, we obtain the black solid curve. To avoid confusion, we refer to this mean profile as the pair of coherent vortices and denote it by $\tilde{\omega}$, while the name condensate vortices is kept for $\bar{\omega}$ [see

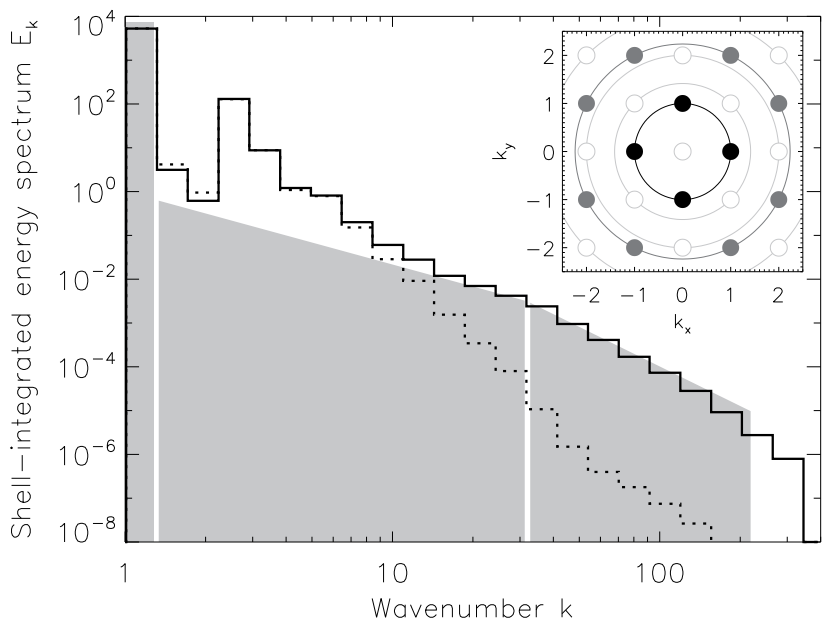

FIG. 8: The solid and dotted histogram are the energy spectra $E_{k}$ of the full $(\omega)$ and the coherent $(\tilde{\omega})$ vorticity in simulation c32 at $t=10000$. The spectral bins have even width in log-scale. The valley and second peak right next to the condensate mode are physical. The valley corresponds to the $|\boldsymbol{k}|=\sqrt{2} k_{1}$ and $2 k_{1}$ modes, which have even $k_{x}+k_{y}$ (open circles in inset). The second peak corresponds to the $|\boldsymbol{k}|=\sqrt{5} k_{1}$ modes, which are the first harmonics of the condensate (gray circles in inset).

definition (21)]. The function $\tilde{\omega}(x, 0)$ can be well approximated by a sharp Gaussian core (fitted by the dashed curve) and exponential wings (fitted by the dotted curve) as shown in the figure. There is no preference between positive and negative vorticity so $\tilde{\omega}$ must change sign in the domain, which implies faster than exponential fall off for $|x| \sim L / 2$. This non-trivial shape cannot be fully described by the 4 modes with $|\boldsymbol{k}|=k_{1}$.

Nevertheless, it is possible to describe $\tilde{\omega}$ by a small number of Fourier coefficients. We find the Fourier coefficients of the coherent vortices satisfying $\left|\tilde{\omega}_{\boldsymbol{k}}\right| \gtrsim 10^{-3}\left|\tilde{\omega}_{k_{1}}\right|$ and list the "upper triangular" part in Table II. The shown values are real because of Hermitian symmetry. The lower triangle and other quadrants are given by $\tilde{\omega}_{k_{x}, k_{y}}=\tilde{\omega}_{ \pm k_{y}, \pm k_{x}}$ because of parity and discrete rotational symmetry. The open circles in Fig. 7 show the reconstructed profile from these coefficients. Despite the fact that we only have a few modes, the approximation is extremely good in both the exponential wings and the far tails where the power falls off more rapidly. The sharp Gaussian core, however, requires higher wave numbers to be represented correctly because of its short length scale.

The omitted values in the table exhibit an interesting pattern. The Fourier coefficients with even $k_{x}+k_{y}$ are significantly smaller than the ones with odd $k_{x}+k_{y}$. This is because the coherent vortices follow the same symmetry properties of the condensate,

$$
\underbrace{\tilde{\omega}(x, y)=\overbrace{\tilde{\omega}(x+L, y+L)}^{\text {flip sign along diagonals }}=-\tilde{\omega}(x+L / 2, y+L / 2)}_{\text {strictly periodic }} .
$$




\begin{tabular}{c|ccccccccccc}
\hline \hline$k_{x}$ & & & & & \multicolumn{1}{c}{$k_{y}$} & & & & & \\
& 0 & 1 & 2 & 3 & 4 & 5 & 6 & 7 & 8 & 9 & 10 \\
\hline 0 & & 28.38 & & 5.03 & & 1.65 & & 0.79 & & 0.45 & \\
1 & & & 10.37 & & 2.24 & & 1.05 & & 0.61 & & 0.32 \\
2 & & & & 2.54 & & 1.43 & & 0.72 & & 0.41 & \\
3 & & & & & 1.75 & & 0.8 & & 0.52 & & 0.14 \\
4 & & & & & & 0.85 & & 0.6 & & 0.32 & \\
5 & & & & & & & 0.64 & & 0.38 & & \\
6 & & & & & & & & 0.4 & & & \\
\hline \hline
\end{tabular}

TABLE II: The "upper triangular" part of the Fourier coefficients of the coherent vortices, which are real because of Hermitian symmetry. The lower triangle and other quadrants are given by $\tilde{\omega}_{k_{x}, k_{y}}=\tilde{\omega}_{ \pm k_{y}, \pm k_{x}}$ because of parity and discrete rotational symmetry. Coefficients that do not satisfy $\left|\tilde{\omega}_{\boldsymbol{k}}\right| \gtrsim 10^{-3}\left|\tilde{\omega}_{k_{1}}\right|$ are omitted in the table. We can reconstruct the shape of both the exponential wings and fast fall off in Fig. 7 (see open circles in the figure) by using the shown coefficients. The sharp Gaussian core, however, requires higher wave numbers to be represented correctly. The omitted values exhibit an interesting pattern that can be derived by symmetry, see Sec. III C for details.

Fourier transforming the above equation, we immediately obtain the condition

$$
\tilde{\omega}_{k_{x}, k_{y}}=-\tilde{\omega}_{k_{x}, k_{y}}(-1)^{k_{x}+k_{y}}
$$

Hence, the coherent vortices can only occupy modes with odd $k_{x}+k_{y}$.

The above property has interesting implication for the energy spectrum of $2 \mathrm{D}$ turbulence. We graphically represent the Fourier amplitudes in the inset of Fig. 8. The most energetic $k_{1}$ modes, which correspond to the value 28.38 in Table II, are marked with filled circles. Their "higher harmonics", with value 10.37, are in dark gray, which form the second peak with radius $\sqrt{5} k_{1}$ in the integrated spectrum as shown by the solid histogram. Other omitted modes with even $k_{x}+k_{y}$ are marked as open circles, which form a spectral valley in the spectrum.

The spectral valley and the second peak next to the condensate in Fig. 8 are, to our knowledge, not seen in previous numerical studies such as $[5-7,11]$. It is possible that these earlier studies have not reached the late saturated stage we study, or perhaps because evenly spacing bins "wash" out the spectra as in Fig. 3. In the later case, reducing the bin size to $k_{1} / 2$ should make the spectral valley and second peak visible, although the spectrum may become noisier. The spectrum obtained from atmospheric data [22] or from direct numerical simulations with Ekman friction [23] would also not have these features as in those cases energy from the large scales is removed by friction. Nevertheless, we should remark that in Fig. 3 of Hossain et al. [4], there is an indication of such a second peak, although the authors did not comment on it.

As we show by the symmetry argument, the non-trivial shape of the coherent vortices is responsible for these additional spectral features. To verify this, we plot the energy spectrum of $\tilde{\omega}$ by a dashed histogram in Fig. 8 . The tight agreement between the solid and dotted histograms in small wave number strongly support our claim.

\section{DISCUSSION}

In this paper, we use a three-scale model to derive the saturation time scales and saturation levels of the condensate and turbulent fluctuation in 2D hydrodynamic turbulence. This requires integrating the 2D NavierStokes equations for a very long time. This has been made possible by virtue of the high performance of the GPUs. We use the Ornstein-Uhlenbeck process as a phenomenological model to describe the movement of condensate vortices. In terms of the saturation time scales $\tau_{\bar{E}}$ and $\tau_{Z^{\prime}}$, the DNS agree quite well with the analytical predictions. The saturation levels are, however, off by a constant factor $\zeta=0.76$. We speculate that this disagreement is because our three-scale model ignores the non-trivial shape of the coherent vortices. The higher harmonics of the condensate modify the spectra at small wave number and affect the saturation level. We have checked that it is not possible to remedy this problem by just changing $k_{1}$ in our model to an effective wave number $k_{\text {cond }} \gtrsim k_{1}$. A more sophisticated model is needed.

Before we conclude, we should point out that the direct (forward) enstrophy flux,

$$
\eta_{\text {dir }} \equiv \eta_{\infty} \approx f_{\mathrm{i}}^{2}\left(k_{\mathrm{i}}^{2}-k_{1}^{2}\right),
$$

is smaller than the enstrophy input $f_{\mathrm{i}}^{2} k_{\mathrm{i}}^{2}$ in our threescale model. There is no inconsistency here. Their difference is simply the (very small) inverse enstrophy leakage

$$
\eta_{\mathrm{inv}} \equiv f_{\mathrm{i}}^{2} k_{\mathrm{i}}^{2}-\eta_{\mathrm{dir}} \approx f_{\mathrm{i}}^{2} k_{1}^{2}
$$

that we commented on for Eq. (9). The property $\eta_{\text {inv }} \ll$ $\eta_{\text {dir }}$ is nothing magical. It is simply a consequence of the broken power law in our three-scale model.

Similarly, we can check how the direct (forward) energy leakage $\epsilon_{\text {dir }}$ compares with the inverse cascade $\epsilon_{\text {inv }}$. Recalling that $\bar{\epsilon}$ is only the inverse energy transfer rate 
at $k_{1}$, we need to add the contribution from $2 \nu Z^{\prime}$,

$$
\begin{aligned}
& \epsilon_{\mathrm{inv}} \equiv \bar{\epsilon}+3 \nu \gamma Z_{\infty}^{\prime} \approx f_{\mathrm{i}}^{2}\left[1-\left(1-\frac{3}{2} \gamma\right) \frac{k_{\mathrm{i}}^{2}}{\gamma k_{\mathrm{d}}^{2}}\right], \\
& \epsilon_{\mathrm{dir}} \equiv 4 \nu \gamma \ln \frac{k_{\mathrm{d}}}{k_{\mathrm{i}}} Z_{\infty}^{\prime} \approx f_{\mathrm{i}}^{2}\left(1-\frac{3}{2} \gamma\right) \frac{k_{\mathrm{i}}^{2}}{\gamma k_{\mathrm{d}}^{2}} .
\end{aligned}
$$

Using the original definition (20), it is easy to verify that $1-3 \gamma / 2 \lesssim 1$, which allows us to conclude $\epsilon_{\text {inv }} \gg \epsilon_{\text {dir }}$. Most of the input energy is inversely cascaded and dissipated at the condensate. Note that the above approximations are made at the same order. The fact that $\epsilon_{\mathrm{inv}}+\epsilon_{\mathrm{dir}}=f_{\mathrm{i}}^{2}$ holds, therefore, shows the consistence of our model.

Finally, our work stresses the fact that, as in threedimensional turbulence [24], the order of taking the limit of long time $(t \rightarrow \infty)$ and small viscosity $(\nu \rightarrow 0)$ is also crucial in 2D turbulence. Specifically, taking the limit $\nu \rightarrow 0$ first leads to a (linear) divergence in time

$$
\lim _{t \rightarrow \infty}\left(\lim _{\nu \rightarrow 0} \bar{E}\right)=\lim _{t \rightarrow \infty} f_{\mathrm{i}}^{2} t .
$$

We must take the limits in the correct order, which is,

$$
\begin{aligned}
\lim _{\nu \rightarrow 0}\left(\lim _{t \rightarrow \infty} \bar{E}\right) & =\lim _{\nu \rightarrow 0}\left(\frac{f_{\mathrm{i}}^{2}}{2 \nu k_{1}^{2}} \frac{\gamma k_{\mathrm{d}}^{2}-k_{\mathrm{i}}^{2}}{\gamma k_{\mathrm{d}}^{2}-k_{1}^{2}}\right) \\
& =\lim _{\nu \rightarrow 0} \frac{f_{\mathrm{i}}^{2}}{2 \nu k_{1}^{2}} .
\end{aligned}
$$

The system can then reach a steady state as long as the viscosity remains non-zero, even without the Ekman term.

\section{Acknowledgments}

All simulations presented in this paper were done on the Zorn cluster in PDC and the Platon cluster in Lunarc. CKC is supported by a NORDITA fellowship. CKC and AB thank John Bowman and Malcolm Roberts for insightful discussions, which motivated this work. All three authors thank Alex Hubbard and two anonymous referees who helped improve the paper. We also thank the organizers of the Nature of Turbulence workshop and are grateful to the hospitality by UCSB and KITP. Financial support from European Research Council under the AstroDyn Research Project 227952 and the Swedish Research Council under the grant 2011-5423 are gratefully acknowledged.
[1] R. H. Kraichnan, Physics of Fluids 10, 1417 (1967).

[2] C. E. Leith, Physics of Fluids 11, 671 (1968).

[3] G. K. Batchelor, Physics of Fluids 12, 233 (1969).

[4] M. Hossain, W. H. Matthaeus, and D. Montgomery, Journal of Plasma Physics 30, 479 (1983).

[5] L. M. Smith and V. Yakhot, Physical Review Letters 71, 352 (1993).

[6] L. M. Smith and V. Yakhot, Journal of Fluid Mechanics 274, 115 (1994).

[7] M. Chertkov, C. Connaughton, I. Kolokolov, and V. Lebedev, Physical Review Letters 99, 084501 (2007), arXiv:nlin/0612052.

[8] J. Paret and P. Tabeling, Physics of Fluids 10, 3126 (1998).

[9] H. Xia, H. Punzmann, G. Falkovich, and M. G. Shats, Physical Review Letters 101, 194504 (2008), 0806.3116.

[10] G. Eyink, Physica D 91, 97 (1996).

[11] C. V. Tran and J. C. Bowman, Phys. Rev. E 69, 036303 (2004).

[12] A. Brandenburg, Astrophys. J. 550, 824 (2001), arXiv:astro-ph/0006186.

[13] J. H. Williamson, Journal of Computational Physics 35, 48 (1980).

[14] D. J. Higham, SIAM Review 43, 525 (2001).
[15] See Supplemental Material at [URL will be inserted by publisher] for a movie showing that the condensate vortex wanders around randomly inside the computational domain.

[16] U. Frisch, Z. S. She, and P. L. Sulem, Physica D Nonlinear Phenomena 28, 382 (1987).

[17] P. L. Sulem, Z. S. She, H. Scholl, and U. Frisch, Journal of Fluid Mechanics 205, 341 (1989).

[18] A. Brandenburg and B. V. Rekowski, A\&A 379, 1153 (2001), arXiv:astro-ph/0106280.

[19] G. E. Uhlenbeck and L. S. Ornstein, Physical Review 36, 823 (1930).

[20] S. Chandrasekhar, Reviews of Modern Physics 15, 1 (1943).

[21] M. C. Wang and G. E. Uhlenbeck, Reviews of Modern Physics 17, 323 (1945).

[22] K. S. Gage and G. D. Nastrom, Radio Science 20, 1339 (1985).

[23] P. Perlekar, S. S. Ray, D. Mitra, and R. Pandit, Physical Review Letters 106, 054501 (2011), 1009.1494.

[24] U. Frisch, Turbulence: The legacy of A. N. Kolmogorov (Cambridge University Press, 1995). 